Max-Planck-Institut für demografische Forschung

Max Planck Institute for Demographic Research

Konrad-Zuse-Strasse 1 - D-18057 Rostock · GERMANY

Tel +49 (0) 3812081 - 0; Fax +49 (0) 3812081 - 202;

http://www.demogr.mpg.de

MPIDR WORKING PAPER WP 2005-033

NOVEMBER 2005

Müttererwerbstätigkeit in Ost- und

Westdeutschland

Eine Analyse mit den Mikrozensen

1991-2002

Esther Geisler (geisler@ demogr.mpg.de)

Michaela Kreyenfeld (kreyenfeld@ demogr.mpg.de)

This working paper has been approved for release by: Gerda Ruth Neyer (neyer@ demogr.mpg.de)

Deputy Head of the Laboratory of Contemporary European Fertility and Family Dynamics.

(C) Copyright is held by the authors.

Working papers of the Max Planck Institute for Demographic Research receive only limited review. Views or opinions expressed in working papers are attributable to the authors and do not necessarily reflect those of the Institute. 


\title{
Müttererwerbstätigkeit in Ost- und Westdeutschland Eine Analyse mit den Mikrozensen 1991-2002
}

\author{
Esther Geisler und Michaela Kreyenfeld ${ }^{\star}$
}

Zusammenfassung: Auf Basis der Daten des Mikrozensus aus den Jahren 1991, 1996 und 2002 gibt dieser Artikel einen Überblick über das Erwerbsverhalten von Frauen mit Kindern in Ost- und Westdeutschland. Neben der Frage der Ost-WestAngleichung stehen sozialstrukturelle Unterschiede im Erwerbsverhalten im Vordergrund der Analyse. Wir argumentieren insbesondere, dass die familien- und sozialpolitischen Rahmenbedingungen in Deutschland eine Zunahme der sozialstrukturellen Unterschiede in der Erwerbsbeteiligung forciert haben.

Stichwörter: Frauenerwerbstätigkeit, Mikrozensus, ILO-Erwerbskonzept

Korrespondenzadresse: Max-Planck-Institut für demografische Forschung, Konrad-Zuse Str. 1, 18057 Rostock. Telefon: +49-381-2081-136 Email: geisler@demogr.mpg.de; kreyenfeld@demogr.mpg.de. 


\section{$1 \quad$ Einleitung}

Ähnlich wie in anderen ehemals sozialistischen Ländern gehörte eine hohe Frauenerwerbsquote zu den expliziten Zielen der Sozialpolitik der DDR, die durch umfassende Maßnahmen nicht nur gefördert, sondern auch gefordert wurde. Im Gegensatz dazu gelten die sozialpolitischen Rahmenbedingungen der BRD als „familialistisch“, d.h. sie zielen weniger auf die Vereinbarkeit Kind von Beruf, sondern fördern vielmehr die Pflege- und Betreuungsarbeit in der Familie (Gauthier 1996; Gornick, Meyers und Ross 1998; Esping-Andersen 1999; Stier, Lewin-Epstein und Braun 2001; Leitner, Ostner und Schratzenstaller 2004).

Mit der Wiedervereinigung im Jahr 1990 wurden die politischen und ökonomischen Institutionen der DDR durch das System der BRD ersetzt. Während in der DDR die Vollzeiterwerbstätigkeit von Frauen mit Kindern eine Selbstverständlichkeit darstellte, bieten die neuen sozialpolitischen Rahmenbedingungen größere Handlungsspielräume. Der (Teil-)Rückzug aus dem Arbeitsmarkt, um sich der Betreuung der Kinder zu widmen, ist nun durch die im internationalen Vergleich großzügige Förderung des „Hausfrauenmodells“ möglich. Vor diesem Hintergrund ist nicht nur mit einer Annäherung der ostdeutschen an die westdeutschen Erwerbsmuster $\mathrm{zu}$ rechnen, auch sollten die sozialstrukturellen Unterschiede in der Erwerbsbeteiligung im Laufe der Zeit zugenommen haben. Zum einen schließt die Arbeitsmarktentwicklung verstärkt schlecht qualifizierte Frauen aus dem primären Arbeitsmarkt aus. Zum anderen setzen die familien- und sozialpolitischen Rahmenbedingungen in Deutschland die niedrigsten Erwerbsanreize für schlecht qualifizierte Frauen.

Ziel dieses Artikels ist es, einen Überblick über die Entwicklung des Erwerbsverhaltens von Müttern in Ost- und Westdeutschland zu geben. Ein Schwerpunkt liegt auf der Frage, welchen Einfluss sozialstrukturelle Faktoren auf die Vollzeiterwerbstätigkeit von Frauen mit Kindern haben und inwiefern diese Faktoren sich im Laufe der 1990er Jahre verändert haben. Der Artikel ist in vier Teile gegliedert. In Abschnitt 2 liefern wir einen Überblick über die familienpolitischen Rahmenbedingungen in Ost- und Westdeutschland vor und nach der Wende. Auf dieser Basis generieren wir Hypothesen zu den sozialstrukturellen Determinanten des Erwerbsverhaltens. Teil 3 beinhaltet einen deskriptiven Überblick über das 
Erwerbsverhalten von Müttern in den Jahren 1991 bis 2002. Als Datenbasis dient der Mikrozensus. In diesem Teil diskutieren wir das standardmäßig verwendete ILOErwerbskonzept und schlagen ein „erweitertes Konzept“ vor, das geeigneter erscheint, das Erwerbsverhalten von Frauen mit Kindern abzubilden. In Abschnitt 4 stellen wir die Ergebnisse eines logistischen Regressionsmodells $\mathrm{zu}$ den sozialstrukturellen Determinanten der Vollzeiterwerbstätigkeit vor.

\section{Familienpolitische Rahmenbedingungen und die Erwerbstätigkeit von Frauen in der DDR und BRD}

\subsection{Familienpolitik in der DDR}

Die Integration von Frauen in den Arbeitsmarkt war eine zentrale sozialpolitische Leitvorstellung der DDR. Da hohe Geburtenraten ebenso hoch auf der politischen Agenda standen, zählten zu den sozialpolitischen Maßnahmen umfassende Regelungen zur Vereinbarkeit von Kind und Beruf (Gysi und Speigner 1983; Koch und Knöbel 1986; Obertreis 1986; Helwig 1988; Cornelius 1990; Frerich und Frey 1993; Trappe 1995; Kreyenfeld 2004). Zu diesen Maßnahmen gehörte insbesondere der Ausbau des Kinderbetreuungssystems, welches flexible und umfassende Ganztagsbetreuungsangebote für Kinder aller Altersklassen bereitstellte. Im Jahr 1989 wurden 80 Prozent der Kinder unter 3 Jahren und 95 Prozent der Kinder im Kindergartenalter institutionell betreut. Die Nutzung der Einrichtungen war abgesehen von geringen Beiträgen für die Verpflegung - kostenlos (Kreyenfeld, Spieß und Wagner 2001: 139). Obwohl der Ausbau der öffentlichen Kinderbetreuung auch dadurch motiviert war, „sozialisationsergänzende Funktionen“ zu erfüllen, war die zentrale Motivation dennoch, Frauen von ihren „häuslichen Pflichten“ zu entlasten (Helwig 1988: 468).

Eine weitere beachtliche sozialpolitische Maßnahme war die Einführung des „Babyjahres“ im Jahr 1976, welches Müttern ab der Geburt ihres zweiten Kindes eine bezahlte Freistellung bot. ${ }^{1}$ Bis dato hatte die Vorstellung dominiert, dass Frauen 
problemlos in den Erwerbsprozess integriert werden konnten. Kritische Studien zu den Langzeiteffekten der Krippenbetreuung sowie die niedrigen Geburtenraten der 1970er hatten im Wesentlichen eine Neukonzeption der Politik motiviert, die es Frauen ermöglichte, sich für ein Jahr der Betreuung ihrer Kinder zu widmen. Das Babyjahr war, wie andere familienpolitische Leistungen auch, auf Mütter fokussiert. Prinzipiell sollten die sozialpolitischen Rahmenbedingungen zwar Frauen bei der Vereinbarkeit von Kind und Beruf unterstützen; allerdings wurde der Mann kaum aufgefordert, sich an der Kindererziehung oder an der Hausarbeit zu beteiligen. Letztendlich blieb die Kinderbetreuung in der Zuständigkeit der Frauen, während sich an den traditionellen Geschlechterrollen wenig veränderte (Helwig 1988: 474; Penrose 1990: 63; Pascall und Manning 2000: 254).

Obwohl das Ziel der Geschlechtergleichheit nicht erreicht wurde, näherten sich die Erwerbsverläufe von Frauen und Männer in der DDR an (Trappe 1995; Trappe und Rosenfeld 1998; Trappe und Rosenfeld 2000). Das Erwerbsverhalten ostdeutscher Frauen zeichnete sich durch eine, zumeist nur durch das Babyjahr unterbrochene, kontinuierliche Vollzeiterwerbstätigkeit aus. Die Einkommensunterschiede zwischen Männern und Frauen waren relativ gering. Insbesondere im Vergleich zur Situation in der BRD waren Frauen in der DDR deutlich häufiger in Führungspositionen zu finden. Das flexible und flächendeckende Betreuungssystem, das Überangebot an offenen Stellen, sowie ein moralischer und ökonomischer Druck machten eine Vollzeiterwerbstätigkeit - für Männer wie für Frauen - zur gesellschaftlichen Normalität.

\subsection{Familienpolitik in der BRD}

In der international vergleichenden Wohlfahrtsstaatsforschung wird die BRD häufig als Prototyp des „familialistischen“ Wohlfahrtsstaates bezeichnet (Gauthier 1996; Gornick, Meyers und Ross 1998; Esping-Andersen 1999; Stier, Lewin-Epstein und Braun 2001; Leitner, Ostner und Schratzenstaller 2004). Demnach fördern die institutionellen Rahmenbedingungen in Westdeutschland einseitig die Einbindung der Frau in Pflege- und Betreuungsarbeit innerhalb des Haushalts, während Maßnahmen 
zur Integration von Frauen in den Arbeitsmarkt eine nur spärliche Beachtung finden. Die Kernelemente des Systems sind das „Ehegattensplitting“, die relativ lange Dauer der Elternzeit und das geringe Angebot an Ganztagsbetreuungsplätzen. Diese Kernelemente sollen im Folgenden kurz dargestellt werden.

Die Dauer des Erziehungsurlaubs (bzw. der Elternzeit) ist seit der Einführung im Jahr 1986 vielfach modifiziert worden. Aktuell sind Frauen und Männer nach der Geburt eines Kindes berechtigt, Erziehungsurlaub für die Dauer von drei Jahren in Anspruch zu nehmen. Für maximal zwei der drei Jahre kann ein einkommensabhängiges Erziehungsgeld von 300 Euro bezogen werden. ${ }^{2}$ In Bezug auf das sozialpolitische Ziel der „Vereinbarkeit von Kind und Beruf“ sind die Regelungen zum Erziehungsurlaub und zum Erziehungsgeld ambivalent beurteilt worden (Gottschall und Bird 2003; Ziefle 2004; Gruescu und Rürup 2005). Zwar wird gleichermaßen Müttern und Vätern die Möglichkeit eingeräumt, für einige Zeit aus dem Beruf auszusteigen, um sich der Betreuung ihrer Kinder zu widmen. Andererseits stellt das Erziehungsgeld von monatlich 300 Euro keine Lohnersatzleistung dar. Nach der Aussage des Bundesfamilienministeriums ist eine Lohnersatzleistung, die bspw. in Schweden mit dem Erziehungsgeld durchaus verbunden ist, mit den deutschen Regelungen nicht angestrebt. Stattdessen soll das Erziehungsgeld als Familienleistung zur „Anerkennung für die besonders wichtige persönliche Betreuung des Kindes in seinen ersten Lebensjahren" dienen (BMFSFJ 2003: 7). Der unzureichende Charakter einer Lohnersatzleistung dürfte jedoch der wesentliche Grund sein, warum der Erziehungsurlaub für Väter bis heute unattraktiv ist.

Die Regelungen zum Erziehungsurlaub können jedoch kaum die niedrigen Müttererwerbstätigenquoten in Westdeutschland erklären. Wichtiger erscheint hier, dass diese Regelungen in ein System implementiert sind, in dem Kinderbetreuungseinrichtungen zwar vorhanden sind, aber bislang keine wesentliche Lösung für Vereinbarkeitsprobleme darstellen. Seit den 1970er Jahren ist die Anzahl der Kindergartenplätze in Westdeutschland kontinuierlich angestiegen. Motiviert war der Ausbau der Kinderbetreuung durch die Zielvorstellungen, Kinder frühzeitig zu fördern und ihnen möglichst ähnliche Startbedingungen zu gewährleisten. Die Frage

Seit dem 1.1.2004 wurde die Einkommensgrenze für die ersten sechs Lebensmonate des Kindes deutlich gesenkt. Sie liegt zurzeit bei 30.000 Euro für Ehepaare und nichteheliche Lebensgemeinschaften sowie 23.000 Euro für Alleinerziehende. Ab 2004 wurde auch die Höhe des Erziehungsgeldes geringfügig gekürzt. So beträgt der Regelbetrag gegenwärtig 300 Euro, bei verkürzter Bezugsdauer werden 450 Euro gezahlt (BMSFJ 2005). 
der „Vereinbarkeit von Kind und Beruf“" stand zu dieser Zeit nicht im Vordergrund. Aus diesem Grund ist es auch nicht verwunderlich, dass sich der Kindergarten nur als eine Halbtagsbetreuungsinstitution etablierte. Überraschenderweise hat sich an dieser Ausrichtung bis heute nur wenig verändert. Zwar wurde 1996 ein gesetzlich verankerter Anspruch auf einen Kindergartenplatz eingeführt, jedoch bezieht sich auch dieser nur auf einen Halbtagsplatz. Ganztagsbetreuungsplätze stellen weiterhin eine Ausnahme dar. Auch die Betreuungssituation für Kinder unter 3 Jahren hat sich in Westdeutschland seit den 1970er Jahren kaum verändert. Die Versorgungsquote liegt weiterhin bei unter 5 Prozent (Statistisches Bundesamt 2004b).

Die Regelungen zum Erziehungsurlaub und das geringe Angebot an Ganztagsbetreuungsplätzen für Kinder sind eingebettet in steuer- und transferpolitische Rahmenbedingungen, die das „Hausfrauenmodell“ ermöglichen. Das „Ehegattensplitting“ erlaubt verheirateten Paaren, ihre Steuern gemeinsam zu veranlagen. Auf Grund des progressiven Steuertarifs bieten sich die größten Steuervorteile für Paare, die das klassische „male breadwinner model“ leben oder solche, bei denen die Frau lediglich einen geringen Beitrag zum Haushaltseinkommen in Form von Teilzeitarbeit leistet (Seidel, Teichmann und Thiede 1999; Dingeldey 2002). Auch die Hinterbliebenenversorgung und die Mitversicherung der nichterwerbstätigen Ehefrau in der gesetzlichen Krankenversicherung sind wichtige Elemente in einem System, in dem die finanziellen Risiken der reduzierten Erwerbstätigkeit von verheirateten Frauen sozial abgefedert werden. Internationale Studien, die den Vergleich sozialpolitischer Rahmenbedingungen zum Kern haben, kommen meist $\mathrm{zu}$ dem Schluss, dass in kaum einem anderen Land die Nichterwerbstätigkeit verheirateter Frauen stärker gefördert wird als durch das System der BRD (Sainsbury 1997).

\subsection{Familienpolitik und Erwerbsverhalten nach der Wende: Anpassung an ein familialistisches Wohlfahrtsregime?}

Mit der Wiedervereinigung im Jahr 1990 wurden die politischen und ökonomischen Institutionen der DDR durch das System der BRD ersetzt. Vor dem Hintergrund der Konvergenz der institutionellen Rahmenbedingungen wurde langfristig eine Annäherung des Erwerbsverhaltens ostdeutscher Frauen an die westdeutschen 
Erwerbsmuster erwartet (Dorbritz 1997; Rosenfeld, Trappe und Gornick 2004). Dies würde bedeuten, dass ostdeutsche Frauen nicht mehr dem „,dual earner model“ folgen würden, sondern dem „,modernisierten male breadwinner model“ (Pfau-Effinger 1999; Klement und Rudolph 2004), in dem nur der Mann Vollzeit erwerbstätig ist und die Frau nach der kindbedingten Erwerbsunterbrechung in die Teilzeiterwerbstätigkeit zurückkehrt.

Prinzipiell scheint es einleuchtend $\mathrm{zu}$ sein, dass sich, angesichts des Institutionentransfers, das Verhalten in Ostdeutschland dem Verhalten in Westdeutschland langfristig annähert. Jedoch ist gerade die Frage, durch welche Mechanismen diese Konvergenz stattfindet, von entscheidendem Interesse. Ein genauerer Blick auf die anhaltenden Unterschiede in den neuen und alten Bundesländern erscheint gerade deshalb sinnvoll, weil es Einsicht in die Frage des Zusammenspiels von Präferenzen, Rahmenbedingungen und Verhalten geben kann. Die Anpassungshypothese stellt letztendlich auch die westdeutschen Verhaltensmuster auf den Prüfstand. Insbesondere wirft sie die Frage auf, inwiefern die westdeutschen Verhaltensmuster überhaupt einen Fixpunkt für einen Vergleich darstellen können und welchem Wandel sie selbst unterworfen sind.

Während im Bereich der ökonomischen Entwicklung und des Arbeitsmarkts eine Angleichung des Ostens an die Bedingungen in den westlichen Bundesländern mit einer Verbesserung der Lebensbedingungen gleichzusetzen ist, ist mehr als fraglich, ob diese Perspektive auch für die Familienentwicklung und die Erwerbstätigkeit von Frauen passend ist. Westdeutschland zeichnet sich durch einen einmalig hohen Anteil zeitlebens kinderloser Frauen aus (Dorbritz und Schwarz 1996). Diejenigen, die sich für Kinder entscheiden, folgen einem Erwerbsmodell, das in der international vergleichenden Wohlfahrtsstaatsforschung dafür kritisiert worden ist, dass es Frauen in die Abhängigkeit des „männlichen Ernährers“ bringt (Orloff 1993; Ostner 1995; Langan und Ostner 1991; Lewis und Ostner 1994). In diesem Sinne ist die Abkehr vom Vollzeiterwerbsmuster nicht mit einer graduellen Änderung im Erwerbsverhalten, sondern mit einer Abkehr von der ökonomischen Eigenständigkeit ostdeutscher Frauen mit Kindern gleichzusetzen. 
Was die Hypothese der Ost-West-Anpassung verkennt, ist, dass es markante Unterschiede in den Rahmenbedingungen und in den Einstellungen gibt, die, gegen jede Erwartungen, die Wende ,überlebt“ haben. Diese Unterschiede betreffen insbesondere die Rahmenbedingungen zur Vereinbarkeit von Kind und Beruf, aber auch grundlegende Einstellungen zur Erwerbstätigkeit von Frauen mit Kindern.

Die sozialpolitischen Bemühungen der DDR, die auf die Integration von Frauen in den Arbeitsmarkt zielten, hatten prinzipiell wenig an der traditionellen Arbeitsteilung im Haushalt verändert. Jedoch hat die Politik durchgreifende Änderungen in den Einstellungen zur Frauenerwerbstätigkeit befördert, die auch die Wende überdauert haben. Dies lässt sich u.a. an den Äußerungen zur Frage, ob ein Kleinkind darunter leidet, wenn seine Mutter erwerbstätig ist, erkennen. Der Vergleich der Analysen 1992 und 2004 zeigt, dass in beiden Landesteilen die liberalen Einstellungen in diesem Zeitraum deutlich zugenommen haben. Während im Jahr 2004 allerdings 62 Prozent der westdeutschen Befragten dieser Einschätzung zustimmen, sind es im Osten gerade mal 29 Prozent (Tabelle 1). In Bezug auf die Einstellungen zur Müttererwerbstätigkeit hat demnach keine Ost-West-Angleichung stattgefunden. Auch zeigen diese Analysen, dass die westdeutschen Einstellungen zur Müttererwerbstätigkeit Veränderungen unterworfen sind.

Tabelle 1: Einstellungen zur Müttererwerbstätigkeit in Deutschland 1992-2004 (Bewertung der Aussage: „Ein Kleinkind wird sicherlich darunter leiden, wenn seine Mutter berufstätig ist.“) (Spaltenprozente)

\begin{tabular}{|l|cccc|cccc|}
\hline & \multicolumn{4}{|c|}{ Westdeutschland } & \multicolumn{4}{c|}{ Ostdeutschland } \\
& 1992 & 1996 & 2000 & 2004 & 1992 & 1996 & 2000 & 2004 \\
\hline Stimme voll und ganz zu & 45 & 43 & 38 & 31 & 23 & 20 & 13 & 8 \\
Stimme eher zu & 29 & 31 & 32 & 31 & 28 & 28 & 27 & 21 \\
Stimme eher nicht zu & 16 & 16 & 19 & 25 & 26 & 27 & 33 & 36 \\
Stimme gar nicht zu & 8 & 8 & 9 & 12 & 21 & 23 & 24 & 34 \\
Weiß nicht & 3 & 2 & 2 & 1 & 3 & 2 & 2 & 1 \\
Insgesamt & 100 & 100 & 100 & 100 & 100 & 100 & 100 & 100 \\
\hline
\end{tabular}

Quelle: ALLBUS 1992, 1996, 2000, 2004 (eigene Berechnungen) 
Der wohl entscheidende Parameter für die Unterstützung der Vereinbarkeit von Kind und Beruf ist das Kinderbetreuungssystem. Obwohl das Angebot an Krippenplätzen nach der Wende deutlich reduziert worden ist, kam es nicht, wie vielfach angenommen, $\mathrm{zu}$ einem „Zusammenbruch“ der ostdeutschen Betreuungsinfrastruktur (Rindfuss und Brewster 1996: 273; Adler 1997: 44). Im Jahr 2002 werden 35 Prozent der Kinder zwischen 0 und unter 3 Jahren in der Krippe betreut (Tabelle 2). In Westdeutschland sind es gerade mal 5 Prozent der Kinder derselben Altersklasse. Ein wesentlicher Grund, warum es nicht zur Angleichung der Betreuungsinfrastruktur an die Bedingungen der alten Länder gekommen ist, mag sein, dass Kinderbetreuung im Wesentlichen in kommunale Zuständigkeit fällt. Ostdeutsche Kommunen haben (bislang) - trotz erheblicher Sparzwänge - diese Infrastrukturleistungen aufrechterhalten (Deutsches Jugendinstitut 2002; Hank, Kreyenfeld und Spieß 2004). Letztendlich ermöglichen die relativ guten Kinderbetreuungsmöglichkeiten in Ostdeutschland weiterhin ein „dual earner model“, wenngleich die steuer- und transferpolitischen Maßnahmen andere Erwerbskonstellationen fördern.

Tabelle 2: Betreuungsquoten in Kindertageseinrichtungen in Deutschland ${ }^{3}$

\begin{tabular}{|l|ccc|ccc|}
\hline & \multicolumn{3}{|c|}{ Westdeutschland } & \multicolumn{3}{c|}{ Ostdeutschland } \\
Alter der Kinder & 1991 & 1996 & 2002 & 1991 & 1996 & 2002 \\
\hline 0 bis unter 3 Jahre & 4 & 4 & 5 & 58 & 33 & 35 \\
3 bis unter 4 Jahre & 51 & 54 & 68 & 89 & 85 & 86 \\
5 bis unter 6 Jahre & 75 & 76 & 77 & 88 & 85 & 86 \\
\hline
\end{tabular}

Anmerkung: Betreuungsquoten: Anzahl der Kinder in einer Kindertageseinrichtung pro 100 Kinder der jeweiligen Altersklasse

Quelle: Mikrozensus 1991, 1996, 2002 (eigene Berechnungen)

Für Kinder im Hortalter ist es auf Grund von Veränderungen im Frageprogramm des Mikrozensus nicht möglich, vergleichbare Analysen für die Jahre 1991 bis 2002 darzustellen. Auch wird nicht der Umfang der Betreuung (ganztags oder halbtags) erfasst. 


\section{Sozialstrukturelle Unterschiede im Erwerbsverhalten}

Inwiefern das „dual earner model“ realisiert wird, hängt jedoch nicht zuletzt von den Arbeitsmarktbedingungen ab. Auf der einen Seite spricht die schlechte Arbeitsmarktentwicklung dafür, dass das ,male breadwinner model“, welches auf der Idee einer stabilen Erwerbskarriere des männlichen Ernährers fußt, für die Mehrzahl der ostdeutschen Paare unattraktiv ist. Auf der anderen Seite drängt die Arbeitsmarktentwicklung schlecht qualifizierte Frauen in Arbeitslosigkeit ab oder verweist sie auf ABM-Maßnahmen (Holst und Kirner 1995; Engelbrech und Reinberg 1997).

Die unterschiedlichen Erwerbschancen von Frauen mit niedrigem und hohem Qualifikationsniveau mögen sozialstrukturelle Unterschiede im Erwerbsverhalten begründen. Ein anderer wesentlicher Aspekt, der sozialstrukturelle Unterschiede im Erwerbsverhalten befördern mag, sind die steuer- und transferpolitischen Maßnahmen, von denen insbesondere Frauen mit geringen Einkommen profitieren. Während das Erziehungsgeld in Ländern wie Schweden vom vorhergehenden Einkommen abhängt und somit für alle Personen ähnliche Anreize setzt, es in Anspruch zu nehmen, ist das Erziehungsgeld in Deutschland nicht an das vorhergehende Einkommen gebunden und beträgt generell 300 Euro. Für Frauen mit einem niedrigen Einkommen sind in Bezug auf Erwerbsentscheidungen derartige Transferleistungen bedeutsamer als für Frauen mit einem hohen Einkommen. Auch das „Ehegattensplitting“ setzt die höchsten „work disincentives“ für Frauen mit niedrigem Einkommen, vorausgesetzt sie haben einen Partner, der ein entsprechend höheres Einkommen erwirtschaftet.

Ähnliche Steuerungswirkungen lassen sich der Höhe der Elternbeiträge, die für die Nutzung von Kindertageseinrichtungen erhoben werden, beimessen. Insbesondere seit der Einführung des Rechtsanspruchs auf einen Kindergartenplatz kam es in den meisten Bundesländern und Kommunen zu einer Anhebung der Elternbeiträge. Während im Jahr 1996 ein Vollzeitplatz in einer Kindertageseinrichtung in Deutschland durchschnittlich 140 DM (70 Euro) gekostet hat, sind es im Jahr 2002 etwa 100 Euro. ${ }^{4}$ Prinzipiell sollen die Elternbeiträge einkommensabhängig gestaltet werden (KJHG § 8), jedoch gehen die einzelnen

Die Angaben beziehen sich auf eigene Berechnungen mit dem Sozio-Oekonomischen Panel. Es handelt sich hier um ungewichtete deskriptive Analysen der Variabeln zum Besuch einer Kindertageseinrichtung und der Höhe der Elternbeiträge. 
Bundesländer und Kommunen sehr unterschiedlich mit dieser gesetzlichen Vorgabe um. Hohe Elternbeiträge können relevante Entscheidungsparameter für das Erwerbsverhalten von Frauen darstellen. Während gut qualifizierte Frauen mit hohem Einkommen eher in der Lage sind, Elternbeiträge zu entrichten, können diese Kosten ein entscheidendes Hemmnis für die Erwerbsaufnahme schlecht qualifizierter Frauen darstellen.

Vor dem Hintergrund dieser Rahmenbedingungen lässt sich vermuten, dass die sozialstrukturellen Unterschiede in der Erwerbsbeteiligung von Müttern im Laufe der 1990er Jahre zugenommen haben. Ein Schwerpunkt der folgenden Analyse liegt auf den sozialstrukturellen Determinanten der Vollzeiterwerbstätigkeit. Wir konzentrieren uns auf die Vollzeiterwerbstätigkeit, da dieser in der international vergleichenden Wohlfahrtsstaatsforschung eine zentrale Rolle für die Eigenständigkeit von Frauen beigemessen wird.

\section{Deskriptive Analysen}

\subsection{Datensatz und Stichprobenauswahl}

Für die empirischen Analysen verwenden wir die Daten des Mikrozensus. Der Mikrozensus ist eine repräsentative Ein-Prozent-Stichprobe der in Deutschland lebenden Bevölkerung. Die Befragung wird seit 1957 einmal jährlich in Westdeutschland durchgeführt. In Ostdeutschland fand die erste MikrozensusBefragung nach der Wende im Jahr 1991 statt. Seit 2005 wird der Mikrozensus auch unterjährig durchgeführt, d.h. ab diesem Zeitpunkt liegen auch Quartalsergebnisse vor. Für die Wissenschaft wird vom Statistischen Bundesamt ein „Scientific-UseFile“ des Mikrozensus zur Verfügung gestellt. Der Scientific-Use-File ist eine faktisch-anonymisierte 70-Prozent-Teilstichprobe des Mikrozensus (Emmerling und Riede 1997; Schimpl-Neimanns 1998). Bislang sind faktisch-anonymisierte Teildatensätze für die Mikrozensen bis zum Jahr 2003 verfügbar. 
Für die folgenden Analyen verwenden wir die Scientfic-Use-Files der Mikrozensen der Jahre 1991, 1996 und 2002..$^{5}$ Der Vorteil des Mikrozensus liegt insbesondere in seiner hohen Fallzahl, so dass auch spezielle Populationen wie in der vorliegenden Untersuchung differenziert analysiert werden können. Darüber hinaus bietet der Mikrozensus den Vorteil, dass über die Jahre hinweg ein relativ vergleichbares Frageprogramm verwendet wird, so dass Veränderungen über die Zeit abgebildet werden können. Das Frageprogramm des Mikrozensus wird gesetzlich verabschiedet und ist für mehrere Jahre festgelegt. Somit ist das Frageprogramm des Mikrozensus 1996 und 2002 fast deckungsgleich. Da der Mikrozensus 1991 noch unter einem anderen Gesetzentwurf fiel, weichen teilweise die Frageformulierungen ab, was die Analysemöglichkeiten einschränkt. Um beispielsweise eine Vergleichbarkeit der Mikrozensen 1996 und 2002 mit dem Mikrozensus 1991 zu gewährleisten, verwenden wir das traditionelle Konzept der Lebensformen, wodurch es nur möglich ist, die Partnermerkmale von verheirateten Frauen, jedoch nicht jene von Frauen in nichtehelichen Lebensgemeinschaften zu berücksichtigen.

In die Analyse beziehen wir nur Frauen zwischen 18 und 45 Jahren ein, die der Bevölkerung am Familienwohnsitz zugerechnet werden können und Kinder unter 15 Jahren haben, die im Haushalt (bzw. in der Familie) leben. Wir verwenden nur Frauen bis zum Alter 45, da wir davon ausgehen können, dass deren Kinder das elterliche Haus in den meisten Fällen noch nicht verlassen haben. Dies ist relevant, da die Fertilitätsbiographie im Mikrozensus nicht abgefragt wird und demzufolge nur Informationen über die im Haushalt bzw. in der Familie lebenden Kinder berücksichtigt werden können. Für jedes Jahr stehen uns mehr als 28.000 westdeutsche Mütter zur Verfügung. In Ostdeutschland nehmen auf Grund des Geburtenrückgangs nach der Wende die Fallzahlen über die 1990er Jahre ab. Stehen uns im Jahr 1991 noch fast 10.000 Frauen mit Kindern zur Analyse zur Verfügung, sind es 2002 nur noch etwa 5.000 (siehe Tabelle A3 im Anhang).

Beim Mikrozensus handelt es sich um eine rotierende Stichprobe, in der die Haushalte jedes Auswahlbezirks vier Jahre lang befragt werden, wobei jedes Jahr ein Viertel der Auswahlbezirke ausgetauscht wird. Für die folgenden Analysen werden die unterschiedlichen Jahre in einem Datensatz gepoolt. Um zu gewährleisten, dass eine befragte Person nicht mehrfach in dem Datensatz erscheint, haben wir die Mikrozensen so ausgewählt, dass zwischen ihnen mindestens vier Jahre Abstand bestehen. 


\subsection{Das ,,erweiterte“ Erwerbskonzept zur Erfassung der}

\section{Müttererwerbstätigkeit}

Eine gängige Kennziffer zur Erfassung des Erwerbsverhaltens von Frauen ist die Erwerbsquote, die den Anteil der Erwerbspersonen an der Wohnbevölkerung wiedergibt. Die Erwerbsquote wird auf Basis der Arbeitskräftestichproben in den Ländern Europas nach den Richtlinien der International Labor Organization (ILO) generiert und in unterschiedlichen Veröffentlichungen der OECD verbreitet (OECD 2005). ${ }^{6}$ In Deutschland ist die Arbeitskräftestichprobe in den Mikrozensus integriert, der die wesentlichen Fragen enthält, um die ILO-Erwerbsquote umzusetzen (Schmidt 2000; Rengers 2004). Demnach liegt die Erwerbsquote von Frauen in Deutschland mit etwa 65 Prozent im Jahr 2002 im europäischen Mittelfeld.

Dass Deutschland, angesichts seiner Rahmenbedingungen, die allgemein als wenig „work and family friendly“ gelten, derart „gut“ im internationalen Vergleich abschneidet, dürfte nicht nur der Tatsache geschuldet sein, dass Ost- und Westdeutschland, spätestens seit 2000, in den europäischen Veröffentlichungen nicht mehr getrennt erscheinen. Während in Ostdeutschland die Erwerbsquote von Frauen bei 73 Prozent, und damit mit den skandinavischen Ländern am oberen Ende der europäischen Verteilung zu finden ist, liegt Westdeutschland mit 64 Prozent deutlich niedriger (siehe Tabelle A1 und A2 im Anhang).

Die Erwerbsquote von Frauen wird vielfach als Indikator verwendet, um die Position von Frauen und die Verwirklichung der Geschlechtergleichheit auf dem Arbeitsmarkt darzustellen. Problematisch erscheint daran, dass sich die Frage, inwiefern die Rahmenbedingungen „,work and family friendly“ sind, in erster Linie nach der Familiengründung stellen. Deutschland (West) zeichnet sich durch ein im internationalen Vergleich hohes Alter bei Erstgeburt und einen extrem hohen Anteil

Die ILO-Definition fasst alle Personen über 15 Jahren unter die Erwerbstätigen, die in der Berichtswoche in einem Beschäftigungsverhältnis stehen, das mindestens 1 Stunde in der Woche umfasst. Weiterhin gelten auch Personen, die zwar in einem Beschäftigungsverhältnis stehen, in der Berichtswoche aber nicht gearbeitet haben, als erwerbstätig.

Als Erwerbslose gelten laut ILO-Definition Personen über 15 Jahre, die in der Berichtswoche keiner entgeltlichen Tätigkeit nachgehen, in den letzten vier Wochen davor aktiv eine Erwerbstätigkeit gesucht haben sowie innerhalb der nächsten zwei Wochen für den Arbeitsmarkt verfügbar sind. Weiterhin zählen auch Personen, die die Arbeitssuche bereits abgeschlossen haben und später als in der Berichtswoche eine Tätigkeit aufnehmen, zu den Erwerbslosen.

Nach dem ILO-Konzept gelten alle Personen als Nichterwerbspersonen, die in der Berichtswoche in keinem Beschäftigungsverhältnis standen und auch nicht auf der Suche nach einer Beschäftigung waren. 
zeitlebens kinderloser Frauen aus. Für die internationale Vergleichbarkeit der Erwerbsquote bedeutet dies, dass in Deutschland das Erwerbsverhalten von kinderlosen Frauen überproportional Berücksichtigung findet. Ein Indikator, der etwas über die Verwirklichung der Geschlechtergleichheit auf dem Arbeitsmarkt, die Vereinbarkeit von Kind und Beruf und die Verwirklichung der „ökonomischen Eigenständigkeit von Frauen“ aussagen will, müsste sich insbesondere auf das Erwerbsverhalten von Frauen mit Kindern beziehen.

Das ILO-Erwerbskonzept, auf dessen Basis die Erwerbsquoten berechnet werden, ist jedoch für die Analyse der Müttererwerbstätigkeit in Ost- und Westdeutschland nicht uneingeschränkt brauchbar. Zum einen werden Frauen im Erziehungsurlaub seit 1996 explizit als Erwerbstätige eingestuft. Zwar lässt sich argumentieren, dass Frauen im Erziehungsurlaub einen gültigen Arbeitsvertrag haben, jedoch sind sie de facto nicht in den Erwerbsprozess integriert. Sinnvoller erscheint es, diese Personen zu der Gruppe der Nichterwerbspersonen zu rechnen (für eine ähnliche Herangehensweise, siehe Dathe 1999; John und Stutzer 2002). Zum anderen unterscheidet das ILO-Erwerbskonzept nicht zwischen Vollzeit, Teilzeit und geringfügig Erwerbstätigen. Insbesondere, wenn es um die Frage der „ökonomischen Eigenständigkeit“"geht, erscheint es wichtig, Vollzeit erwerbstätige und andere Frauen zu unterscheiden.

Vor diesem Hintergrund haben wir für die folgenden Analysen der Müttererwerbstätigkeit ein „erweitertes“ Erwerbskonzept verwendet. Vorrangiges Anliegen ist dabei, Personen im Erziehungsurlaub zu den Nichterwerbspersonen zu rechnen. Darüber hinaus unterscheiden wir nach dem Erwerbsumfang. Vollzeiterwerbstätige sind Personen, die für die Berichtswoche eine tatsächliche Arbeitszeit von mindestens 30 Stunden angeben. ${ }^{7}$ Teilzeiterwerbstätige sind Personen mit einer Arbeitszeit von 15 bis 29 Stunden, geringfügig Beschäftigte sind Personen mit einem Wochenarbeitsumfang von 1 bis 14 Stunden. Erwerbslose sind (analog zur

\footnotetext{
Zur Abgrenzung der Vollzeiterwerbstätigkeit verwenden wir entgegen der bislang gebräuchlichen Grenze von 35 Stunden und mehr Arbeitszeit pro Woche die 30-StundenGrenze. Auf diese Weise erfolgt die Abgrenzung analog zu der seit 1997 verwendeten OECDDefinition, die berücksichtigt, dass in einigen Sektoren die vertragsgemäße Vollzeiterwerbstätigkeit bei 35 Stunden oder sogar etwas darunter liegen kann (Bastelaer et al. 1997). In den „Demographischen Standards“ des Statistischen Bundesamtes (2004a: 4) sind die Empfehlungen zur Abgrenzung der Vollzeiterwerbstätigkeit eher vage formuliert: „Mit diesen einerseits erweiterten und andererseits nicht mehr über eine exakte Arbeitszeit definierten Kategorien wird den neuen Entwicklungen auf dem Arbeitsmarkt Rechnung getragen, denn die alten Grenzen (35 Stunden und mehr als ,vollzeit', 15 bis 34 stunden als ,teilzeit' und unter 15 Stunden als ,stundenweise' erwerbstätig) gelten nicht mehr."
} 
ILO-Klassifikation) Personen, die nicht erwerbstätig sind, auf Arbeitssuche sind und dem Arbeitsmarkt sofort zur Verfügung stehen. ${ }^{8}$ Nichterwerbspersonen sind (ebenfalls analog zur ILO-Klassifikation) Personen, die in keinem Beschäftigungsverhältnis stehen und nicht auf der Suche nach einer Beschäftigung sind. Darüber hinaus haben wir in diese Gruppe die Personen im Erziehungsurlaub hinzugefügt. ${ }^{9}$

\subsection{Deskriptive Darstellung: Der Rückgang der Vollzeiterwerbstätigkeit seit der Wende}

Auf Basis des „erweiterten Erwerbskonzepts“ haben wir in Tabelle 3 das Erwerbsverhalten von Müttern in Ost- und Westdeutschland nach dem Alter des jüngsten Kindes dargestellt. Für Ostdeutschland ist insbesondere ein Anstieg der Erwerbslosen, Teilzeit und geringfügig Erwerbstätigen erkennbar. Demgegenüber ist der Anteil der Vollzeitzeiterwerbstätigkeit deutlich rückläufig. Während im Jahr 1992 noch 60 Prozent der ostdeutschen Mütter Vollzeit erwerbstätig waren, sind es im Jahr 2002 nur noch knapp 50 Prozent. Trotz dieses Rückgangs ist die Vollzeiterwerbstätigenquote in den neuen Bundesländern mehr als dreimal so hoch wie in den alten Bundesländern.

Betrachtet man die Erwerbsquoten nach dem Alter des jüngsten Kindes, zeigt sich in Ostdeutschland insbesondere für Frau mit Kindern unter 3 Jahren ein

$8 \quad$ Auf Grund der Veränderung des Frageprogramms bezieht sich die Verfügbarkeit im Jahr 1991 auf eine „,sofortige Verfügbarkeit“. Im Mikrozensus 1996 und 2002 bezieht sich diese auf eine Verfügbarkeit ,innerhalb von zwei Wochen“.

9 Erst ab dem Mikrozensus 1999 wird explizit nach dem Erziehungsurlaub gefragt. Wir grenzen aus diesem Grund Personen im Erziehungsurlaub darüber ein, dass sie für die Berichtswoche eine Arbeitzeit von „null Stunden“ angeben, obwohl sie in einem Arbeitsverhältnis stehen. Der Nachteil an dieser Klassifikation ist, dass man durch dieses Vorgehen auch Personen, die auf Grund von Krankheit in der Berichtswoche nicht erwerbstätig sind, in die Kategorie der Nichterwerbspersonen einordnet. Jedoch sind die weite Mehrzahl der Personen in dieser Kategorie Personen im Erziehungsurlaub. Wir verwenden die ,tatsächlich“ geleistete Arbeitszeit und nicht die ,normalerweise“ geleistet Arbeitszeit, da unklar ist, welche Arbeitszeiten Personen angeben, die sich im Erziehungsurlaub befinden. Wir verwenden ebenfalls nicht die Variable zur Vollzeit-Teilzeiterwerbstätigkeit, auf dessen Basis sich Personen selbst einstufen können, da zum einen auch hier nicht klar ist, zu welcher Kategorie sich Erziehungsurlauberinnen rechnen und es des Weiteren aufgrund der fehlenden Erläuterung der Begriffe Voll- und Teilzeiterwerbstätigkeit zu Fehleinschätzungen der Befragten kommt. Am Rande sei angemerkt, dass eine Analyse dieser Variable einen leichten Anstieg der Vollzeiterwerbstätigkeit der Frauen mit Kindern in Westdeutschland ergibt. 
deutlicher Anstieg der Nichterwerbspersonen. ${ }^{10}$ Im Jahr 1991 lag in dieser Gruppe der Anteil der Nichterwerbspersonen bei 41 Prozent und stieg bis zum Jahr 1996 auf 60 Prozent. Diese Entwicklung mag mit der Verlängerung des Erziehungsurlaubs, der 1992 von 18 Monaten auf drei Jahre ausgeweitet wurde, in Zusammenhang stehen. In den anderen Gruppen liegt der Anteil der „Hausfrauen“ seit der Wende jedoch bei weniger als 15 Prozent. ${ }^{11}$

In Westdeutschland hat zwischen 1991 und 2002 der Anteil der Teilzeiterwerbstätigen und geringfügig Beschäftigten stark zugenommen, während der Anteil der „Hausfrauen“ kontinuierlich zurückgegangen ist. Überraschenderweise ist die Vollzeiterwerbstätigenquote seit dem Jahr 1992 von einem niedrigen Anfangsniveau von 19 Prozent weiter auf 16 Prozent im Jahr 2002 zurückgegangen. Diese Entwicklung könnte prinzipiell mit der Verlängerung des Erziehungsurlaubs im Jahr 1992 in Verbindung gebracht werden. Gegen eine derartige Interpretation spricht jedoch, dass die Vollzeiterwerbstätigkeit für Frauen mit Kindern aller Altersgruppen gleichermaßen zurückgegangen ist, d.h. auch für Frauen, die keinen Anspruch auf Erziehungsurlaub haben. Insgesamt zeigen diese Entwicklungen, dass die steigende Erwerbsquote in Westdeutschland vor allem auf die Zunahme der Teilzeit- und geringfügigen Beschäftigung zurückzuführen ist.

Der Rückgang der Vollzeiterwerbstätigkeit lässt sich nur schwerlich in die internationalen Entwicklungen einordnen. Die meisten Länder haben im Laufe der letzen Jahre bzw. Jahrzehnte sozialpolitische Maßnahmen implementiert, die die Vereinbarkeit von Familien und Beruf unterstützen und die Integration von Frauen in den Arbeitsmarkt fördern. Vor diesem Hintergrund wurde vielfach die Erwartung geäußert, dass es einen stetigen Trend gibt, in welchem die Frauenerwerbstätigkeit,

Der relativ hohe Anteil von Nichterwerbspersonen in den neuen Bundesländern im Jahr 1991 ist darauf zurückzuführen, dass unter die Nichterwerbspersonen auch Personen fallen, die einen Arbeitsvertrag haben, sofern sie in der Berichtswoche eine tatsächliche Arbeitszeit von ,null Stunden“ angegeben haben. In Westdeutschland sind dies in erster Linie Frauen im Erziehungsurlaub, die in diese Gruppe fallen. Auf Grund der transformationsbedingten Arbeitsmarktentwicklung befinden sich in dieser Gruppe jedoch im Jahr 1991 in Ostdeutschland in erster Linie Personen, die vorübergehend von ihrer Arbeit freigestellt worden waren. Im Mikrozensus geben die meisten ostdeutschen Befragten, die wir im Jahr 1991 als Nichterwerbspersonen klassifiziert haben an, dass sie in „Kurzarbeit“ beschäftigt sind.

11 Am Rande sei angemerkt, dass unter den ostdeutschen Nichterwerbspersonen häufiger Personen sind, die noch im Studium sind. Im Jahr 2002 trifft dies auf etwas mehr als 3 Prozent der ostdeutschen Mütter zu, in den alten Bundesländern auf weniger als 2 Prozent. Ein Vergleich des Anteils von Müttern in Ausbildung ist für die unterschiedlichen Jahre leider nicht möglich, da die Fragen zur Bildungsbeteiligung aus dem Jahr 1991 und den Jahren 1996/2002 nicht vergleichbar sind. 
die ökonomische Eigenständigkeit und die Geschlechtergleichheit zunimmt. Daly (2005) argumentiert in diesem Zusammenhang: „The movement in participation levels is inexorably upwards. While a disappearance of the housewife might be premature, increased employment among women is associated with a move towards two-income families and a decline in the male breadwinnerl female homemaker household arrangement" (ebd.: 383). Zwar lässt sich in Westdeutschland ein deutlicher Rückgang des „female homemaker“-Anteils konstatieren, jedoch wird diese Entwicklung auch von einem leichten Rückgang der Vollzeiterwerbstätigkeit begleitet.

Tabelle 3: Erwerbsbeteiligung nach Alter des jüngsten Kindes (Spaltenprozente)

\begin{tabular}{|c|c|c|c|c|c|c|c|c|c|}
\hline & \multicolumn{3}{|c|}{ Ostdeutschland } & \multicolumn{3}{|c|}{ Westdeutschland } & \multicolumn{3}{|c|}{ Deutschland } \\
\hline & 1991 & 1996 & 2002 & 1991 & 1996 & 2002 & 1991 & 1996 & 2002 \\
\hline $\begin{array}{l}\text { Alle Frauen mit Kindern } \\
\text {-- Vollzeit }\end{array}$ & 60,1 & 58,1 & 47,9 & 18,5 & 16,1 & 15,6 & 29,1 & 24,6 & 20,4 \\
\hline -- Teilzeit & 7,8 & 7,6 & 9,3 & 18,5 & 18,3 & 21,2 & 15,8 & 16,2 & 19,4 \\
\hline -- geringfügig & 0,8 & 1,4 & 3,6 & 7,0 & 9,9 & 14,6 & 5,4 & 8,2 & 12,9 \\
\hline -- erwerbslos & 10,3 & 16,8 & 15,6 & 3,1 & 3,8 & 3,8 & 4,9 & 6,4 & 5,6 \\
\hline -- Nichterwerbsperson & 21,0 & 16,2 & 23,6 & 52,9 & 51,8 & 44,9 & 44,7 & 44,6 & 41,7 \\
\hline $\begin{array}{l}\text { Jüngstes Kind } 0 \text { - unter } 3 \\
\text {-- Vollzeit }\end{array}$ & 410 & 204 & 245 & 103 & 82 & 78 & 164 & 95 & 10 \\
\hline -- Teilzeit & 5,6 & 5,2 & 6,5 & 10,8 & 7,2 & 8,0 & 9,8 & 7,0 & 7,8 \\
\hline -- geringfügig & 0,7 & 2,1 & 3,6 & 5,3 & 6,3 & 10,7 & 4,4 & 5,8 & 9,8 \\
\hline -- erwerbslos & 12,1 & 11,9 & 8,3 & 3,0 & 2,1 & 1,5 & 4,8 & 3,2 & 2,4 \\
\hline -- Nichterwerbsperson & 40,7 & 60,4 & 57,1 & 70,7 & 76,2 & 72 & 64,7 & 74,5 & 70,1 \\
\hline Jüngstes Kind 3 - unter 6 & & & & & & & & & \\
\hline $\begin{array}{l}\text {-- Vollzeit } \\
\text {-- Teilzeit }\end{array}$ & $\begin{array}{r}63,9 \\
87\end{array}$ & 55,4 & 50,7 & $\begin{array}{r}18 \\
201\end{array}$ & $\begin{array}{l}14,4 \\
100\end{array}$ & 14,5 & $\begin{array}{l}30,7 \\
170\end{array}$ & 21,1 & $\begin{array}{l}19,2 \\
22,5\end{array}$ \\
\hline $\begin{array}{l}\text {-- Teilzeit } \\
\text {-- geringfügig }\end{array}$ & 8,7 & 8,6 & 10,7 & 20,1 & 19,9 & 24,3 & $\begin{array}{r}17,0 \\
61\end{array}$ & $\begin{array}{l}18,1 \\
105\end{array}$ & $\begin{array}{l}22,5 \\
15,4\end{array}$ \\
\hline -- geringfügig & 1,1 & 1,3 & 3,4 & 8,0 & 12,3 & $\begin{array}{r}17,2 \\
\end{array}$ & $\begin{array}{l}6,1 \\
54\end{array}$ & $\begin{array}{r}10,5 \\
81\end{array}$ & $\begin{array}{r}15,4 \\
7,4\end{array}$ \\
\hline -- erwerbslos & 11,0 & 23,1 & 21,7 & 3,2 & 5,2 & 5,3 & 5,4 & 8,1 & 7,4 \\
\hline -- Nichterwerbsperson & 15,3 & 11,6 & 13,5 & 50,7 & 48,2 & 38,7 & 40,8 & 42,2 & 35,4 \\
\hline Jüngstes Kind 6 - unter 10 & & & & & & & & & \\
\hline$--\mathrm{Voll}$ & 68,3 & 67,8 & 56,7 & 23,3 & 20,1 & 18,1 & 36,2 & 33,6 & 22,7 \\
\hline -- Teilzeit & 8,7 & 8,2 & 11,7 & 25,2 & 26,0 & 29,4 & 20,5 & 20,9 & 27,3 \\
\hline -- geringfügig & 0,8 & 1,2 & 4,4 & 8,6 & 12,9 & 18,0 & 6,4 & 9,6 & 16,4 \\
\hline -- erwerbslos & 9,6 & 16,3 & 18,7 & 3,4 & 4,5 & 4,9 & 5,2 & 7,8 & 6,5 \\
\hline -- Nichterwerbsperson & 12,6 & 6,6 & 8,5 & 39,5 & 36,5 & 29,7 & 31,8 & 28 & 27,2 \\
\hline Jüngstes Kind 10 - unter 15 & & & & & & & & & \\
\hline -- Vollzeit & 71 & 69,6 & 62,7 & 33,2 & 30,8 & 29,4 & 44,6 & 42,4 & 37,6 \\
\hline -- Teilzeit & 8,2 & 7,4 & 9,5 & 25,3 & 28,4 & 31,2 & 20,2 & 22,1 & 25,8 \\
\hline -- geringfügig & 0,6 & 1,3 & 3,3 & 7,1 & 9,4 & 13,3 & 5,2 & 7,0 & 10,8 \\
\hline -- erwerbslos & 7,8 & 15,7 & 16,3 & 2,7 & 4,4 & 5,0 & 4,3 & 7,8 & 7,8 \\
\hline -- Nichterwerbsperson & 12,4 & 6,0 & 8,2 & 31,6 & 27,0 & 21,2 & 25,8 & 20,7 & 18,0 \\
\hline
\end{tabular}

Anmerkung: Vollzeiterwerbstätigkeit: >= 30 h/Woche; Teilzeiterwerbstätigkeit: $15-29$ h/Woche; geringfügige Beschäftigung: <=14 h/Woche

Quelle: Mikrozensen 1991, 1996, 2002 (eigene Berechnungen) 


\section{Sozialstrukturelle Determinanten der Vollzeiterwerbstätigkeit}

\subsection{Methode und Kovariaten}

Mit Hilfe logistischer Regressionsmodelle analysieren wir im Folgenden den Einfluss sozialstruktureller Merkmale auf das Chancenverhältnis, Vollzeit erwerbstätig zu sein. ${ }^{12}$ Die Datensätze der Jahre 1991, 1996 und 2002 werden für die Analysen zusammengefasst. Die Analysen werden für Ost- und Westdeutschland getrennt durchgeführt. Im ersten Schritt schätzen wir ein Modell ohne die Bildung des Partners. Im zweiten Modell beziehen wir diese Kovariate mit ein, wobei diese Information nur für verheiratete Frauen zur Verfügung steht. In einem dritten Schritt untersuchen wir Interaktionseffekte zwischen der Schulbildung und dem Befragungsjahr.

Eine zentrale erklärende Variable ist der Schulabschluss. Wir unterscheiden Befragte mit keinem oder einem Hauptschulabschluss, Befragte mit Realschulabschluss oder einem Abschluss der Polytechnischen Oberschule und Befragte mit Fachhochschulreife oder Abitur. ${ }^{13}$ Weiterhin kontrollieren wir für das Befragungsjahr, d.h. für die Jahre 1991, 1996 und 2002. Das Alter der Frau haben wir in die Kategorien 18 bis 25, 26 bis 30, 31 bis 35 und 36 bis 45 gruppiert. Das Alter des jüngsten Kindes haben wir analog $\mathrm{zu}$ den deskriptiven Analysen in die Kategorien 0 bis unter 3, 3 bis unter 6, 6 bis unter 10 und 10 bis unter 15 Jahre klassifiziert. Darüber hinaus unterschieden wir Frauen nach der Anzahl ihrer Kinder, d.h. ob sie nur ein Kind, zwei Kinder oder drei oder mehr Kinder haben. Da die Fertilitätsbiographie im Mikrozensus nicht erhoben wird, beziehen sich diese Angaben auf die Anzahl der Kinder, die zum Befragungszeitpunkt noch im Haushalt leben. Der Familienstand ist eine weitere zentrale Kontrollvariable. Wir unterscheiden zwischen ledigen und verheirateten Frauen sowie der Gruppe der Geschiedenen und Verwitweten. Für die Gruppe der verheirateten Frauen war es ebenfalls möglich, den

12 Die abhängige Variable im logistischen Regressionsmodell ist der natürliche Logarithmus des Odds, was häufig als „Chancenverhältnis“ übersetzt wird. Da dieser Begriff im deutschen Sprachgebrauch nicht üblich ist, verwenden wir im Folgenden auch die Begriffe „Wahrscheinlichkeit“ und „Chance“. Zwar ist die abhängige Variable im logistischen Regressionsmodell keine Wahrscheinlichkeit, steht aber mit dieser in engem Zusammenhang.

13 Leider ist die Variable zur beruflichen Ausbildung und zum Hochschulabschluss nicht mit den gleichen Antwortvorgaben in den verschiedenen Jahren erhoben worden, weshalb es nicht möglich ist, diese Informationen zu verwenden. 
Schulabschluss des Partners $\mathrm{zu}$ generieren. Die Nationalität ist eine weitere Kontrollvariable, d.h. wir unterscheiden Personen mit deutscher und mit anderer Staatsangehörigkeit.

\subsection{Ergebnisse}

Tabelle 4 gibt die Ergebnisse des multivariaten Modells wieder. Die Ergebnisse zu den Kovariaten Jahr und Alter des ersten Kindes bestätigen im Wesentlichen die deskriptiven Analysen. Es gibt einen kontinuierlichen Rückgang der Vollzeiterwerbstätigkeit über die Zeit. Ist das jüngste Kind jünger als 3 Jahre, ist die Chance einer Vollzeiterwerbstätigkeit sehr gering. In Westdeutschland steigt die Wahrscheinlichkeit der Vollzeiterwerbstätigkeit mit zunehmendem Alter kontinuierlich an. Im Osten gibt es dagegen kaum Unterschiede zwischen Frauen mit Kindern im Grundschulalter (6 bis 10 Jahre) und älteren Kindern (10 bis unter 15 Jahre). Mit steigender Kinderzahl reduziert sich in Ost und West die Chance der Vollzeiterwerbstätigkeit. Die Koeffizienten für das Alter der Mutter zeigen auch in West- und Ostdeutschland ein ähnliches Muster, d.h. die Vollzeiterwerbstätigkeit ist besonders hoch in der Altersklasse 30 bis 35 Jahre.

Die Nationalität hat in den beiden Landesteilen einen unterschiedlichen Einfluss. Während in Westdeutschland Personen mit nicht-deutscher Staatsangehörigkeit eine höhere Vollzeiterwerbsneigung aufweisen als Personen mit deutscher Staatsangehörigkeit, existiert ein gegenläufiger Zusammenhang in den neuen Bundesländern. Für die Interpretation dieses Befundes muss zum einen beachtet werden, dass sich die Herkunftsländer der Ausländerinnen in den neuen und alten Bundesländern deutlich voneinander unterscheidet. Während es sich bei den in Ostdeutschland lebenden Ausländerinnen hauptsächlich um Osteuropäerinnen handelt, die in den 1990er Jahren zugewandert sind, stellen die Türkinnen die größte Gruppe der ausländischen Frauen in Westdeutschland. Prinzipiell haben Ausländerinnen mit Kindern in Westdeutschland eine niedrige Erwerbsneigung (siehe Tabelle A3b). Falls sie jedoch erwerbstätig sind, sind sie ebenso häufig Vollzeit erwerbstätig wie westdeutsche Frauen. Erst nach Kontrolle des Bildungsstatus ergibt sich dann ein positiver Effekt der Nationalität auf die Vollzeiterwerbsbeteiligung. 
Überraschende Ergebnisse liefert der Zusammenhang von Familienstand und Vollzeiterwerbstätigkeit. Vor dem Hintergrund der steuerrechtlichen und sozialversicherungstechnischen Regelungen, die insbesondere verheirateten Frauen zu Gute kommen, würde man vermuten, dass diese seltener Vollzeit erwerbstätig sind als ledige Frauen mit Kindern. Letztere haben nicht die Option auf das „male breadwinner model“" und müssen, sofern sie nicht auf Sozialtransfers angewiesen sein wollen, erwerbstätig sein. In Westdeutschland zeigt sich sehr deutlich, dass verheiratete Frauen seltener Vollzeit erwerbstätig sind als andere. In Ostdeutschland dagegen sind es gerade verheiratete Frauen, die häufiger einer Vollzeiterwerbstätigkeit nachgehen (für eine ausführliche Diskussion dieses Aspekets, siehe auch Konietzka und Kreyenfeld 2005).

Der Schulabschluss der Befragten zeigt den erwarteten Zusammenhang, d.h. mit steigendem Bildungsstand steigt auch die Wahrscheinlichkeit, Vollzeit erwerbstätig zu sein. Dieser Zusammenhang gilt für Ost- und Westdeutschland. Vom Schulabschluss des Partners würde man einen negativen Einfluss erwarten, da sich mit steigender Bildung und mit steigendem Einkommen des Mannes die Möglichkeiten des (Teil-)Rückzugs der Frau aus dem Arbeitsmarkt erhöhen. Dieser Zusammenhang findet sich jedoch nur in Westdeutschland. In Ostdeutschland hat der Schulabschluss des Mannes einen positiven Einfluss auf die Chance der Vollzeiterwerbstätigkeit seiner Partnerin. 
Tabelle 4: Logistisches Regressionsmodell (Odds Ratios), Abhängige Variable: Vollzeit, andere (Referenz)

\begin{tabular}{|c|c|c|c|c|c|}
\hline & & Ostdeı & and & Westde & chland \\
\hline & & Modell 1 & Modell 2 & Modell 1 & Modell 2 \\
\hline Jahr & 1991 & 1 & 1 & 1 & 1 \\
\hline & 1996 & $0,73 * * *$ & $0,73 * * *$ & $0,77 * * *$ & $0,76 * * *$ \\
\hline & 2002 & $0,56 * * *$ & $0,56 * * *$ & $0,68 * * *$ & $0,67 * * *$ \\
\hline Alter & $18-25$ & 1 & 1 & 1 & 1 \\
\hline & $26-30$ & $1,27 * * *$ & $1,27 * * *$ & $1,24 * * *$ & $1,24 * * *$ \\
\hline & $30-35$ & $1,49 * * *$ & $1,49 * * *$ & $1,29 * * *$ & $1,31 * * *$ \\
\hline & $36-45$ & $1,26 * * *$ & $1,21 * * *$ & $1,20 * * *$ & $1,24 * * *$ \\
\hline Jüngstes Kind & 0 - unter 3 & 1 & 1 & 1 & 1 \\
\hline & $3-$ unter 6 & $2,85 * * *$ & $2,86 * * *$ & $1,98 * * *$ & $1,95 * * *$ \\
\hline & 6 - unter 10 & $3,66 * * *$ & $3,67 * * *$ & $2,83 * * *$ & $2,76 * * *$ \\
\hline & 10 - unter 15 & $3,86 * * *$ & $3,88 * * *$ & $4,49 * * *$ & $4,35 * * *$ \\
\hline Anzahl Kinder & Ein Kind & 1 & 1 & 1 & 1 \\
\hline & Zwei Kinder & $0,82 * * *$ & $0,81 * * *$ & $0,59 * * *$ & $0,60 * * *$ \\
\hline & Drei und mehr & $0,42 * * *$ & $0,42 * * *$ & $0,51 * * *$ & $0,51 * * *$ \\
\hline Nationalität & Deutsch & 1 & 1 & 1 & 1 \\
\hline & Andere & $0,29 * * *$ & $0,29 * * *$ & $1,27 * * *$ & $1,26 * * *$ \\
\hline Schulabschluss & Angabe fehlt & $1,83 * * *$ & $1,75 * * *$ & $1,19 * * *$ & $0,88 * *$ \\
\hline & Hauptschule & 1 & 1 & 1 & 1 \\
\hline & Realschule & $1,87 * * *$ & $1,76 * * *$ & $1,20 * * *$ & $1,24 * * *$ \\
\hline & Abitur & $2,95 * * *$ & $2,73 * * *$ & $1,69 * * *$ & $1,86 * * *$ \\
\hline Familienstand & Ledig & $0,79 * * *$ & & $2,38 * * *$ & \\
\hline & Gesch./verw. & $0,88 * *$ & & $2,19 * * *$ & \\
\hline & Verheiratet & 1 & & 1 & \\
\hline Schulabschluss & Ledig & & 0,93 & & $2,35 * * *$ \\
\hline Partner & $\begin{array}{l}\text { Gesch./verw. } \\
\text { Verheiratet }\end{array}$ & & 1,04 & & $2,20 * * *$ \\
\hline & --Angabe fehlt & & 1,14 & & $1,58 * * *$ \\
\hline & --Hauptschule & & 1 & & 1 \\
\hline & --Realschule & & $1,19 * *$ & & 0,97 \\
\hline & --Abitur & & $1,23 * * *$ & & $0,81 * * *$ \\
\hline
\end{tabular}

Anmerkung: Die Stichproben umfassen Frauen im Alter von 18-45 Jahre, die zum Befragungszeitpunkt ein Kind haben, das im Haushalt (bzw. in der Familie) lebt. ***p<0,01;**p<0,05; ${ }^{*}<<0,10$

Quelle: Scientific Use File des Mikrozensus 1991, 1996, 2002 (eigene Berechnungen) 
Die Tabellen 5a und 5b geben die Ergebnisse eines Interaktionsmodells zwischen dem Befragungsjahr und der Schulbildung der Frau wieder. Ziel dieser Interaktion ist es zu zeigen, ob die bildungsspezifischen Unterschiede in den Vollzeiterwerbsmustern zugenommen haben. In Westdeutschland ist unter den Frauen mit Abitur und Realschulabschluss die Vollzeiterwerbsneigung tendenziell konstant geblieben. In Ostdeutschland lässt sich ein leichter Anstieg der Vollzeiterwerbstätigkeit unter den Frauen mit Abitur erkennen. Auffällig ist der starke Rückgang der Vollzeiterwerbstätigkeit unter den Frauen mit Hauptschulabschluss bzw. ohne Abschluss. In Westdeutschland verringerten sich für Frauen mit einem niedrigen Schulabschluss die Chancen, Vollzeit erwerbstätig zu sein, von 1991 auf 2002 um 23 Prozent. ${ }^{14}$ In Ostdeutschland lässt sich ein Rückgang um 30 Prozent konstatieren. Im Gesamtbild ergibt sich daraus eine zunehmende Polarisierung der Vollzeiterwerbstätigkeit nach dem Bildungsstatus der Frau.

Tabelle 5a: Interaktion zwischen dem Jahr und der Schulbildung der Frau. Westdeutschland

\begin{tabular}{|ll|ccc|}
\hline & & \multicolumn{3}{c|}{ Jahr } \\
& & 1991 & 1996 & 2002 \\
\hline Schulabschluss & Hauptschule & 0,98 & $0,84 * * *$ & $0,75 * *$ \\
& Realschule & 1,03 & 1 & 1,04 \\
& Abitur & $1,46 * * *$ & $1,46 * * *$ & $1,43 * * *$ \\
\hline
\end{tabular}

Tabelle 5b: Interaktion zwischen dem Jahr und der Schulbildung der Frau. Ostdeutschland

\begin{tabular}{|ll|ccc|}
\hline & & \multicolumn{3}{c|}{ Jahr } \\
& & 1991 & 1996 & 2002 \\
\hline Schulabschluss & Hauptschule & $0,67 *$ & $0,39 * * *$ & $0,47 * * *$ \\
& Realschule & 1,00 & 1 & 1,09 \\
& Abitur & $1,44 *$ & $1,81 * * *$ & $1,74 * *$ \\
\hline
\end{tabular}

Anmerkung: siehe Tabelle 4. Weitere Variablen im Modell sind Alter der Frau, Alter des jüngsten Kindes. Anzahl der Kinder, Nationalität, Familienstand, Kontrollvariable für fehlende Angaben zum Bildungsabschluss

Quelle: Scientific Use File des Mikrozensus 1991, 1996, 2002 (eigene Berechnungen)

14 Diesen Wert erzielt man, indem man den Effektkoeffizienten aus dem Jahr 2002 durch den im Jahr 1991 dividiert. In diesem Fall wird 0,75 durch 0,98 geteilt. Dies ergibt einen Effektkoeffizienten von 0,77. Entsprechend hat sich das Chancenverhältnis um 23 reduziert. 


\section{$5 \quad$ Schlussfolgerung}

Ziel unserer Darstellungen war es, einen Überblick über die Entwicklung der Müttererwerbstätigkeit auf Basis eines „erweiterten Erwerbskonzepts“ in Ost- und Westdeutschland zu geben. Dieses erweitere Erwerbskonzept klassifiziert - im Unterschied zur ILO-Klassifikation - Frauen im Erziehungsurlaub als Nichterwerbspersonen. Darüber hinaus berücksichtigt es explizit den Erwerbsumfang. Eine Unterscheidung zwischen geringfügig, Teilzeit und Vollzeit erwerbstätig ist zur Analyse des Erwerbsverhaltens von Frauen mit Kindern von besonderer Bedeutung. Im Kontext der vergleichenden Wohlfahrtsstaatsforschung kommt der Kategorie „ökonomische Eigenständigkeit“ eine zentrale Bedeutung zu. Eine ökonomische Eigenständigkeit kann in der Regel nur über eine Vollzeiterwerbstätigkeit erreicht werden. Demnach kommt der Vollzeiterwerbstätigenquote eine zentrale Rolle bei der Beurteilung der Frage der Integration von Frauen in den Arbeitsmarkt zu.

Vergleicht man die ILO-Erwerbsquote mit der Vollzeiterwerbstätigenquote, die wir auf Basis des „erweiterten Konzepts“ berechnet haben, ergeben sich insbesondere für die alten Bundesländer erhebliche Unterschiede. Liegt die ILOErwerbsquote, die standardmäßig für Deutschland ausgewiesen wird, bei 65 Prozent, liegt die Vollzeiterwerbstätigenquote für Mütter in Westdeutschland nur bei etwa 20 Prozent. In Ostdeutschland sind die Unterschiede weniger ausgeprägt. Dort liegt die Vollzeiterwerbstätigenquote bei etwa 50 Prozent. Entsprechend existieren auch mehr als 10 Jahre nach der Wende noch erhebliche Unterschiede im Erwerbsverhalten von Frauen mit Kindern in Ost- und Westdeutschland. Trotz eines deutlichen Rückgangs der Vollzeiterwerbsneigung in den neuen Bundesländern ist bislang keine Anpassung an westdeutsche Verhaltensmuster zu beobachten.

Während in Westdeutschland der Anteil der Frauen, die Teilzeit erwerbstätig sind oder in geringfügigen Beschäftigungsverhältnissen stehen, im Laufe der 1990er Jahre angestiegen ist, ist der Anteil Vollzeiterwerbstätiger leicht zurückgegangen. Die Analysen der sozialstrukturellen Determinanten der Vollzeiterwerbstätigkeit zeigen, dass dieser Rückgang insbesondere die Gruppe der Frauen mit einem niedrigen Bildungsabschluss (Hauptschulabschluss oder ohne Schulabschluss) betroffen hat. Die Polarisierung der Vollzeiterwerbsmuster hat damit in Ost- und Westdeutschland seit der Wende zugenommen. Vollzeiterwerbstätigkeit, und damit ökonomische 
Eigenständigkeit, ist in erster Linie ein Privileg von Müttern mit einem höheren Bildungsabschluss. 


\section{Literatur}

Adler, Marina A. (1997): Social change and decline in marriage and fertility in Eastern Germany. Journal of Marriage and the Family 59 (1): 37-49.

Bastelaer, Alois van / Lemaître; Marianna, Pascal (1997): The definitions of part-time work for the purpose of international comparisons. Labour market and social policy occasional papers 22. Paris: OECD.

BMFSFJ (Hg.) (2003): Erziehungsgeld, Elternzeit: Das neue Bundeserziehungsgeldgesetz für Eltern mit Kindern ab dem Geburtsjahrgang 2001. Berlin: BMFSFJ.

BMFSFJ (Hg.) (2005): Erziehungsgeld, Elternzeit: Das Bundeserziehungsgeldgesetz. Berlin: BMFSFJ.

Cornelius, Ivar (1990): Familien- und Bevölkerungspolitik in der DDR. Arbeit und Sozialpolitik 8/9: 308-316.

Daly, Mary (2005): Changing in family life in Europe: Significance for state and society. European Societies 7 (3): 379-398.

Dathe, Dietmar (1999): Familienlebenszyklus und Erwerbsbeteiligung der Ehepartner in Ehen mit Kindern in West- und Ostdeutschland. In: Lüttinger, Paul (Hg.): Sozialstrukturanalyse mit dem Mikrozensus. Mannheim: ZUMA (ZUMA-Nachrichten spezial 6).

Deutsches Jugendinstitut (DJI) (2002): Zahlenspiegel: Tageseinrichtungen für Kinder. München: Deutsches Jugendinstitut.

Dingeldey, Irene (2002): Das deutsche System der Ehegattenbesteuerung im europäischen Vergleich. WSI Mitteilungen 3: 154-160.

Dorbritz, Jürgen (1997): Der demographische Wandel in Ostdeutschland - Verläufe und Erklärungsansätze. Zeitschrift für Bevölkerungswissenschaft 22 (2-3): 239-268. 
Dorbritz, Jürgen / Schwarz, Karl (1996): Kinderlosigkeit in Deutschland - ein Massenphänomen? Analysen zur Erscheinungsformen und Ursachen. Zeitschrift für Bevölkerungswissenschaft 21(3): 231-261.

Emmerling, Dieter / Riede, Thomas (1997): 40 Jahre Mikrozensus. Wirtschaft und Statistik 3: 160-174.

Engelbrech, Gerhard; Reinberg, Alexander (1997): Frauen und Männer in der Beschäftigungskrise der 90er Jahre: Entwicklung der Erwerbstätigkeit in West und Ost nach Branchen, Berufen und Qualifikationen. IAB Werkstattbericht 11.

Esping-Andersen, Gøsta (1999): Social Foundations of Postindustrial Economies. Oxford: University Press.

Frerich, Johannes / Frey, Martin (1993): Handbuch der Geschichte der Sozialpolitik in Deutschland. Band 2: Sozialpolitik in der Deutschen Demokratischen Republik. München: Oldenbourg Verlag.

Gauthier, Anne H. (1996): The State and the Family. A Comparative Analysis of Family Policies in Industrialized Countries. Oxford: Clarendon Press.

Gornick, Janet C. / Meyers, Marcia K. / Ross, Katherin E. (1998): Public Policies and the Employment of Mothers: A Cross-National Study. Social Science Quarterly 79 (1): $35-54$.

Gottschall, Karin / Bird, Katherine (2003): Family leave policies and labor market segregation in Germany: Reinvention or reform of the male breadwinner model? Review of Policy Research: Special Issue on Gender and Work Place Policies 20(1): 115-134.

Gruescu, Sandra / Rürup, Bert (2005): Nachhaltige Familienpolitik. Aus Politik und Zeitgeschichte 23-24: 3-6.

Gysi, Jutta / Speigner, Wulfram (1983): Changes in the life patterns of families in the German Democratic Republic. Berlin: Academy of Sciences of the GDR. Institute of Sociology and Social Policy. 
Hank, Karsten / Kreyenfeld, Michaela / Spieß, C. Katharina (2004): Childcare and fertility in Germany. Zeitschrift für Soziologie 34 (3): 228-244.

Helwig, Gisela (1988): Staat und Familie in der DDR. In: Glaeßner, Gert-Joachim (Hg.): Die DDR in der Ära Honecker: Politik, Kultur, Gesellschaft. Opladen: Westdeutscher Verlag (Schriften des Zentralinstituts für Sozialwissenschaftliche Forschung der Freien Universität Berlin 56): 466-480.

Holst, Elke / Kirner, Ellen (1995): Ausgrenzung von Frauen in Ostdeutschland nach der "Wende" Maßnahmen zur Verbesserung der Vereinbarkeit von Familie und Beruf. In: Hanesch, Walter (Hg.): Sozialpolitische Strategien gegen Armut. Opladen: Westdeutscher Verlag: 311-325.

John, Birgit / Stutzer, Erich (2002): Erwerbsverhalten von Erziehungsurlauberinnen. Zeitschrift für Familienforschung 3: 215-233.

Klammer, Ute et al. (2000): WSI-FrauenDatenReport. CD-Rom (Beilage zur Buchausgabe WSI FrauenDatenReport). Berlin: ed. Sigma (Forschung aus der HansBöckler-Stiftung 26).

Klement, Carmen / Rudolph, Brigitte (2004): Employment patterns and economic independence of women in intimate relationships. European Societies 6 (3): 299-318.

Koch, Petra/ Knöbel, Hans Günther (1986): Familienpolitik der DDR im Spannungsfeld zwischen Familie und Berufstätigkeit von Frauen. Pfaffenweiler: Centaurus-Verlagsgesellschaft.

Konietzka, Dirk / Kreyenfeld, Michaela (2005): Nichteheliche Mutterschaft und soziale Ungleichheit: zur sozioökonomischen Differenzierung der Familienformen in Ost- und Westdeutschland. Kölner Zeitschrift für Soziologie und Sozialpsychologie 57 (1): 32-61.

Kreyenfeld, Michaela (2004): Fertility decisions in the FRG and GDR: an analysis with data from the German Fertility and Family Survey. Demographic Research S3: 11: 276-318. 
Kreyenfeld, Michaela / Spieß, C. Katharina / Wagner, Gert G. (2001): Finanzierungsund Organisationsmodelle institutioneller Kinderbetreuung: Analysen zum Status quo und Vorschläge zur Reform. Neuwied, Berlin: Luchterhand.

Langan, Mary; Ostner, Ilona (1991): Geschlechterpolitik im Wohlfahrtsstaat: Aspekte im internationalen Vergleich. Kritische Justiz: 302-317.

Leitner, Sigrid / Ostner, Ilona / Schratzenstaller, Margit (2004): Einleitung: Was kommt nach dem Ernährermodell? Sozialpolitik zwischen Re-Kommodifizierung und Re-Familialisierung. In: Dies. (Hg.): Wohlfahrtsstaat und Geschlechterverhältnis im Umbruch: Was kommt nach dem Ernährermodell? Wiesbaden: VS Verlag für Sozialwissenschaften (Jahrbuch für Europa- und Nordamerika-Studien 7): 9-27.

Lewis, Jane; Ostner, Ilona (1994): Gender and the Evolution of European Social Policies. ZeS-Arbeitspapier Nr. 4/94. Bremen: Zentrum für Sozialpolitik.

Obertreis, Gesine (1986): Familienpolitik in der DDR 1945-1980. Opladen: Leske und Budrich.

OECD (2005): Labour Statistics-Datenbank. http://www.oecd.org.

Orloff, Ann (1993): Gender and the social rights of citizenship: The comparative analysis of gender relations and welfare states. American Sociological Review 58: 303-328.

Ostner, Ilona (1995): Arm ohne Ehemann? Sozialpolitische Regulierung von Lebenschancen für Frauen im internationalen Vergleich. Aus Politik und Zeitgeschichte B 36-37: 3-12.

Pascall, Gillian / Manning, Nick (2000): Gender and social policy: comparing welfare states in Central and Eastern Europe and the former Soviet Union. Journal of European Social Policy 10 (3): 240-266.

Penrose, Virginia (1990): Vierzig Jahre SED-Frauenpolitik: Ziele, Strategien und Ergebnisse. Frauenforschung 8: 60-77.

Pfau-Effinger, Birgit (1999): Change of family policies in the socio-cultural context of European societies. Comparative Social Research 18: 135-159. 
Rengers, Martina (2004): Das international vereinbarte Labour-Force-Konzept. Wirtschaft und Statistik 12: 1369-1383.

Rindfuss, Ronald R. / Brewster, Karin L. (1996): Childrearing and fertility. Population and Development Review 22 (Supplement): 258-289.

Rosenfeld, Rachel A. / Trappe, Heike / Gornick, Janet C. (2004): Gender and work in Germany: Before and after unification. Annual Review of Sociology 30: 103-124.

Sainsbury, Diane (1997): Taxation, family responsibilities, and employment. In: Sainsbury, D. (ed.): Gender and Welfare State Regimes. Oxford: Oxford University Press: 185-209.

Schimpl-Neimanns, Bernhard (1998): Analysemöglichkeiten des Mikrozensus. ZUMA-Nachrichten 42: 91-122.

Schmidt, Simone (2000): Erwerbstätigkeit im Mikrozensus: Konzepte, Definition, Umsetzung. Mannheim: ZUMA. ZUMA-Arbeitsbericht 2000/01.

Seidel, Bernhard / Teichmann, Dieter / Thiede, Sabine (1999): Ehegattensplitting nicht mehr zeitgemäß. DIW-Wochenbericht 40, Online-Ausgabe. http://www.diw.de/deutsch/produkte/publikationen/wochenberichte/docs/99-40-

1.html.

Statistisches Bundesamt (2004a): Demographische Standards. Ausgabe 2004. Wiesbaden: Statistisches Bundesamt.

Statistisches Bundesamt (2004b): Sozialleistungen. Tageseinrichtungen für Kinder am 31.12.2002. http://www.destatis.de/basis/d/solei/tab_juhilf.htm.

Statistisches Bundesamt (Hg.) (2003): Bevölkerung und Erwerbstätigkeit. Fachserie 1, Reihe 4.1.1: Stand und Entwicklung der Erwerbstätigkeit 2002. Stuttgart: Metzler Poeschel.

Stier, Haya / Lewin-Epstein, Noah / Braun, Michael (2001): Welfare regimes, familysupportive policies, and women's employment along the life-course. American Journal of Sociology 106: 1731-1760. 
Trappe, Heike (1995): Emanzipation oder Zwang? Frauen in der DDR zwischen Beruf, Familie und Sozialpolitik. Berlin: Akademie Verlag.

Trappe, Heike / Rosenfeld, Rachel A. (1998): A comparison of job-shifting patterns in the former East Germany and the former West Germany. European Sociological Review 14 (4): 343-368.

Trappe, Heike / Rosenfeld, Rachel A. (2000): How do children matter? A comparison of gender earnings inequality for young adults in the former East Germany and the former West Germany. Journal of Marriage and the Family 62 (2): 489-507.

Ziefle, Andrea (2004): Die individuellen Kosten des Erziehungsurlaubs: Eine empirische Analyse der kurz- und längerfristigen Folgen für den Karriereverlauf von Frauen. Zeitschrift für Soziologie und Sozialpsychologie 56 (2): 213-231. 


\section{Anhang}

Tabelle A1: Erwerbsquoten 15-64-jähriger Frauen in europäischen Ländern

\begin{tabular}{|rrrrrrrrrr|}
\hline Jahr & $\begin{array}{c}\text { Däne- } \\
\text { mark }\end{array}$ & $\begin{array}{c}\text { Schwe- } \\
\text { den }\end{array}$ & $\begin{array}{c}\text { Finn- } \\
\text { land }\end{array}$ & $\begin{array}{c}\text { Frank- } \\
\text { reich }\end{array}$ & $\begin{array}{c}\text { Deutsch- } \\
\text { land }\end{array}$ & $\begin{array}{c}\text { Tschech- } \\
\text { ien }\end{array}$ & Ungarn & Italien & Spanien \\
\hline 1991 & 78,9 & 80,2 & 72,7 & 58,2 & 61 & 66,8 & & 46,2 & 42,1 \\
1992 & 79,0 & 78,6 & 71 & 59,0 & 61,3 & 64,1 & 57,6 & 45,7 & 43,0 \\
1993 & 78,3 & 76,7 & 70 & 59,4 & 61,4 & 64,8 & 55 & 42,5 & 43,9 \\
1994 & 73,8 & 75,7 & 69,4 & 60,1 & 61,4 & 65,1 & 52,6 & 42,4 & 45,3 \\
1995 & 73,6 & 75,9 & 70 & 60,3 & 61,5 & 64,9 & 50,1 & 42,8 & 46,2 \\
1996 & 74,1 & 75,8 & 70,3 & 61,0 & 61,9 & 64,4 & 49,6 & 43,5 & 47,1 \\
1997 & 75,1 & 75,0 & 69,6 & 61,3 & 62,5 & 64,4 & 48,8 & 44,1 & 48,2 \\
1998 & 75,2 & 74,2 & 69,9 & 62,1 & 63,1 & 64,7 & 50 & 45,1 & 48,8 \\
1999 & 75,8 & 74,6 & 71,4 & 62,7 & 63,3 & 64,9 & 51,3 & 46,0 & 49,8 \\
2000 & 75,9 & 75,0 & 72,3 & 63,3 & 63,6 & 64,3 & 52,2 & 46,8 & 51,7 \\
2001 & 76,0 & 75,5 & 72,7 & 63,6 & 64,3 & 64 & 52 & 47,8 & 50,4 \\
2002 & 75,6 & 75,6 & 73,1 & 64,2 & 64,6 & 63,7 & 52,4 & 48,4 & 52,0 \\
\hline
\end{tabular}

Quelle: OECD 2005

Tabelle A2: Erwerbsquoten 15-64-jähriger Frauen in West- und Ostdeutschland ${ }^{15}$

\begin{tabular}{|lcc|}
\hline Jahr & Westdeutschland & Ostdeutschland \\
\hline 1991 & 58,4 & 77,2 \\
1992 & 59,5 & 74,8 \\
1993 & 59,6 & 73,3 \\
1994 & 60,0 & 73,8 \\
1995 & 59,9 & 73,9 \\
1996 & 59,7 & 73,4 \\
1997 & 60,3 & 73,6 \\
1998 & 60,5 & 73,5 \\
1999 & 61,7 & 73 \\
2000 & & \\
2001 & 63,2 & 72,5 \\
2002 & 63,6 & 72,6 \\
\hline
\end{tabular}

Quelle: Klammer (2000) und Statistisches Bundesamt (2003)

15 In den Veröffentlichungen der OECD werden die Erwerbsquoten nicht mehr getrennt für Ostund Westdeutschland ausgewiesen. Das Statistische Bundesamt weist weiterhin Erwerbsquoten nach Ost- und Westdeutschland getrennt aus (siehe Tabelle A3). In den Publikationen des Statistischen Bundesamtes werden die Erwerbsquoten jedoch nicht nach ILO-Kriterien ausgewiesen, da das Kriterium der Verfügbarkeit bei den Erwerbslosen nicht mit berücksichtigt wird, sondern alle Erwerbslosen in die Erwerbsquote mit einberechnet werden. Die Frauenerwerbsquote in den Publikationen des Statistischen Bundesamtes liegt deshalb etwas höher als die ILO-Erwerbsquote (um etwa einen Prozentpunkt). Bei Berechnung der Erwerbsquoten mit dem Scientific-Use-File des Mikrozensus nach dem ILO-Konzept ergeben sich entsprechend Abweichungen von etwa einem Prozentpunkt zu den Berechnungen des Statistischen Bundesamts. 
Tabelle A3a: Deskriptive Statistik zum Logistischen Regressionsmodell, Zeilenprozente, Ostdeutschland

\begin{tabular}{|c|c|c|c|c|c|c|c|}
\hline & & Vollzeit & Teilzeit & $\begin{array}{c}\text { Gering- } \\
\text { fügig }\end{array}$ & $\begin{array}{l}\text { werbs- } \\
\text { los }\end{array}$ & Nicht-ewt. & Insgesamt \\
\hline Jahr & 1991 & $60 \%$ & $8 \%$ & $1 \%$ & $10 \%$ & $21 \%$ & $100 \%$ \\
\hline & 1996 & $58 \%$ & $8 \%$ & $1 \%$ & $17 \%$ & $16 \%$ & $100 \%$ \\
\hline & 2002 & $48 \%$ & $9 \%$ & $4 \%$ & $16 \%$ & $24 \%$ & $100 \%$ \\
\hline Alter & $18-25$ & $38 \%$ & $5 \%$ & $1 \%$ & $15 \%$ & $40 \%$ & $100 \%$ \\
\hline & $26-30$ & $53 \%$ & $8 \%$ & $2 \%$ & $14 \%$ & $23 \%$ & $100 \%$ \\
\hline & $30-35$ & $62 \%$ & $8 \%$ & $2 \%$ & $13 \%$ & $15 \%$ & $100 \%$ \\
\hline & $36-45$ & $63 \%$ & $9 \%$ & $2 \%$ & $14 \%$ & $13 \%$ & $100 \%$ \\
\hline Jüngstes Kind & $0-3$ & $32 \%$ & $6 \%$ & $2 \%$ & $11 \%$ & $50 \%$ & $100 \%$ \\
\hline & $3-6$ & $59 \%$ & $9 \%$ & $2 \%$ & $17 \%$ & $14 \%$ & $100 \%$ \\
\hline & $6-10$ & $67 \%$ & $9 \%$ & $2 \%$ & $14 \%$ & $9 \%$ & $100 \%$ \\
\hline & $10-15$ & $68 \%$ & $8 \%$ & $2 \%$ & $13 \%$ & $9 \%$ & $100 \%$ \\
\hline Anzahl Kinder & Ein Kind & $59 \%$ & $8 \%$ & $2 \%$ & $13 \%$ & $18 \%$ & $100 \%$ \\
\hline & Zwei Kinder & $57 \%$ & $9 \%$ & $1 \%$ & $13 \%$ & $19 \%$ & $100 \%$ \\
\hline & Drei + & $36 \%$ & $7 \%$ & $3 \%$ & $17 \%$ & $37 \%$ & $100 \%$ \\
\hline Nationalität & Deutsch & $57 \%$ & $8 \%$ & $2 \%$ & $14 \%$ & $20 \%$ & $100 \%$ \\
\hline & Andere & $24 \%$ & $7 \%$ & $3 \%$ & $22 \%$ & $44 \%$ & $100 \%$ \\
\hline Schulab- & fehlt & $57 \%$ & $9 \%$ & $1 \%$ & $14 \%$ & $19 \%$ & $100 \%$ \\
\hline schluss & Haupt & $38 \%$ & $5 \%$ & $1 \%$ & $25 \%$ & $31 \%$ & $100 \%$ \\
\hline & Real & $56 \%$ & $8 \%$ & $2 \%$ & $15 \%$ & $20 \%$ & $100 \%$ \\
\hline & Abitur & $65 \%$ & $9 \%$ & $2 \%$ & $6 \%$ & $18 \%$ & $100 \%$ \\
\hline Familienstand & Verheiratet & $58 \%$ & $9 \%$ & $2 \%$ & $12 \%$ & $19 \%$ & $100 \%$ \\
\hline & Ledig & $47 \%$ & $6 \%$ & $2 \%$ & $18 \%$ & $28 \%$ & $100 \%$ \\
\hline & Gesch. & $59 \%$ & $6 \%$ & $2 \%$ & $18 \%$ & $15 \%$ & $100 \%$ \\
\hline Schulab- & Ledig & $47 \%$ & $6 \%$ & $2 \%$ & $18 \%$ & $28 \%$ & $100 \%$ \\
\hline schluss & Gesch. & $59 \%$ & $6 \%$ & $2 \%$ & $18 \%$ & $15 \%$ & $100 \%$ \\
\hline Partner & Verheiratet & & & & & & \\
\hline & -- fehlt & $57 \%$ & $8 \%$ & $2 \%$ & $16 \%$ & $16 \%$ & $100 \%$ \\
\hline & -- Haupt & $50 \%$ & $7 \%$ & $1 \%$ & $18 \%$ & $24 \%$ & $100 \%$ \\
\hline & -- Real & $58 \%$ & $9 \%$ & $2 \%$ & $13 \%$ & $18 \%$ & $100 \%$ \\
\hline & -- Abitur & $62 \%$ & $9 \%$ & $1 \%$ & $7 \%$ & $21 \%$ & $100 \%$ \\
\hline Fallzahlen/ & 1991 & 5.897 & 763 & 80 & 1.012 & 2.061 & 9.813 \\
\hline Jahr & 1996 & 4.328 & 565 & 102 & 1.252 & 1.204 & 7.451 \\
\hline & 2002 & 2.374 & 462 & 180 & 773 & 1.169 & 4.958 \\
\hline & Insgesamt & 12.599 & 1.790 & 362 & 3.037 & 4.434 & 22.222 \\
\hline
\end{tabular}

Quelle: Scientific Use File des Mikrozensus 1991, 1996, 2002 (eigene Berechnungen) 
Tabelle A3b: Deskriptive Statistik zum Logistischen Regressionsmodell, Zeilenprozente, Westdeutschland

\begin{tabular}{|c|c|c|c|c|c|c|c|}
\hline & & Vollzeit & Teilzeit & $\begin{array}{c}\text { Gering- } \\
\text { fügig }\end{array}$ & $\begin{array}{l}\text { werbs- } \\
\text { los }\end{array}$ & Nicht-ewt. & Insgesamt \\
\hline \multirow[t]{3}{*}{ Jahr } & 1991 & $19 \%$ & $19 \%$ & $7 \%$ & $3 \%$ & $53 \%$ & $100 \%$ \\
\hline & 1996 & $16 \%$ & $18 \%$ & $10 \%$ & $4 \%$ & $52 \%$ & $100 \%$ \\
\hline & 2002 & $16 \%$ & $21 \%$ & $15 \%$ & $4 \%$ & $45 \%$ & $100 \%$ \\
\hline \multirow[t]{4}{*}{ Alter } & $18-25$ & $11 \%$ & $7 \%$ & $6 \%$ & $4 \%$ & $72 \%$ & $100 \%$ \\
\hline & $26-30$ & $14 \%$ & $13 \%$ & $9 \%$ & $4 \%$ & $60 \%$ & $100 \%$ \\
\hline & $30-35$ & $16 \%$ & $20 \%$ & $11 \%$ & $4 \%$ & $49 \%$ & $100 \%$ \\
\hline & $36-45$ & $20 \%$ & $25 \%$ & $12 \%$ & $3 \%$ & $39 \%$ & $100 \%$ \\
\hline \multirow[t]{4}{*}{ Jüngstes Kind } & $0-3$ & $9 \%$ & $9 \%$ & $7 \%$ & $2 \%$ & $73 \%$ & $100 \%$ \\
\hline & $3-6$ & $16 \%$ & $21 \%$ & $13 \%$ & $5 \%$ & $46 \%$ & $100 \%$ \\
\hline & $6-10$ & $21 \%$ & $27 \%$ & $13 \%$ & $4 \%$ & $34 \%$ & $100 \%$ \\
\hline & $10-15$ & $33 \%$ & $28 \%$ & $10 \%$ & $4 \%$ & $25 \%$ & $100 \%$ \\
\hline \multirow[t]{3}{*}{ Anzahl Kinder } & Ein Kind & $22 \%$ & $21 \%$ & $8 \%$ & $4 \%$ & $44 \%$ & $100 \%$ \\
\hline & Zwei Kinder & $13 \%$ & $20 \%$ & $12 \%$ & $3 \%$ & $52 \%$ & $100 \%$ \\
\hline & Drei + & $10 \%$ & $11 \%$ & $11 \%$ & $3 \%$ & $65 \%$ & $100 \%$ \\
\hline \multirow[t]{2}{*}{ Nationalität } & Deutsch & $17 \%$ & $21 \%$ & $11 \%$ & $3 \%$ & $48 \%$ & $100 \%$ \\
\hline & Andere & $16 \%$ & $9 \%$ & $7 \%$ & $5 \%$ & $62 \%$ & $100 \%$ \\
\hline \multirow{4}{*}{$\begin{array}{l}\text { Schulab- } \\
\text { schluss }\end{array}$} & fehlt & $18 \%$ & $13 \%$ & $6 \%$ & $3 \%$ & $59 \%$ & $100 \%$ \\
\hline & Haupt & $15 \%$ & $16 \%$ & $11 \%$ & $4 \%$ & $54 \%$ & $100 \%$ \\
\hline & Real & $16 \%$ & $23 \%$ & $11 \%$ & $3 \%$ & $46 \%$ & $100 \%$ \\
\hline & Abitur & $20 \%$ & $21 \%$ & $10 \%$ & $3 \%$ & $45 \%$ & $100 \%$ \\
\hline \multirow[t]{3}{*}{ Familienstand } & Verheiratet & $15 \%$ & $19 \%$ & $11 \%$ & $3 \%$ & $52 \%$ & $100 \%$ \\
\hline & Ledig & $30 \%$ & $17 \%$ & $5 \%$ & $7 \%$ & $40 \%$ & $100 \%$ \\
\hline & Gesch. & $34 \%$ & $23 \%$ & $6 \%$ & $8 \%$ & $29 \%$ & $100 \%$ \\
\hline \multirow{7}{*}{$\begin{array}{l}\text { Schulab- } \\
\text { Schluss } \\
\text { Partner }\end{array}$} & Ledig & $30 \%$ & $17 \%$ & $5 \%$ & $7 \%$ & $40 \%$ & $100 \%$ \\
\hline & Gesch. & $34 \%$ & $23 \%$ & $6 \%$ & $8 \%$ & $29 \%$ & $100 \%$ \\
\hline & Verheiratet & & & & & & \\
\hline & -- fehlt & $21 \%$ & $17 \%$ & $8 \%$ & $4 \%$ & $50 \%$ & $100 \%$ \\
\hline & -- Haupt & $14 \%$ & $19 \%$ & $11 \%$ & $3 \%$ & $53 \%$ & $100 \%$ \\
\hline & -- Real & $14 \%$ & $21 \%$ & $12 \%$ & $3 \%$ & $50 \%$ & $100 \%$ \\
\hline & -- Abitur & $14 \%$ & $20 \%$ & $11 \%$ & $3 \%$ & $53 \%$ & $100 \%$ \\
\hline \multirow{4}{*}{$\begin{array}{l}\text { Fallzahlen/ } \\
\text { Jahr }\end{array}$} & 1991 & 5.296 & 5.290 & 1.995 & 877 & 15.132 & 28.590 \\
\hline & 1996 & 4.759 & 5.409 & 2.931 & 1.133 & 15.294 & 29.526 \\
\hline & 2002 & 4.387 & 5.965 & 4.105 & 1.080 & 12.643 & 28.180 \\
\hline & Insgesamt & 14.442 & 16.664 & 9.031 & 3.090 & 43.069 & 86.296 \\
\hline
\end{tabular}

Quelle: Scientific Use File des Mikrozensus 1991, 1996, 2002 (eigene Berechnungen) 